\title{
Introduction to the Special Issue on the Utility of Transdiagnostic Approaches for Developing Novel Interventions for Substance and Behavioural Addictions
}

\author{
Murat Yücel $^{1}$ • Leonardo F. Fontenelle ${ }^{1,2,3}$. Samuel R. Chamberlain ${ }^{4}$
}

Published online: 27 April 2019

(C) Springer Science+Business Media, LLC, part of Springer Nature 2019

It is widely established that certain psychoactive substances (e.g., alcohol, cocaine) affect brain reward pathways including dopamine, opioid, and glutamatergic neurochemical systems (Kalivas \& Volkow, 2005). These biological effects are linked to addiction in some individuals including unsuccessful attempts to reduce intake, narrowing of interests, risky use (continued use despite knowledge of damaging consequences), and physiologic effects (tolerance and withdrawal; American Psychiatric Association, 2013). This special issue recognizes that certain classes of behaviour exert strong influences over these reward pathways and thus are prone to repetition, that is, constitute "behavioural addictions."

This topic is timely, coinciding with the modern recognition of gambling disorder as an addiction (DSM-5; American Psychiatric Association, 2013), but also with the growing evidence that other disorders and syndromes share remarkable parallels and so can be usefully viewed within the addiction framework (Grant, Potenza, Weinstein, \& Gorelick, 2010; Mueller et al., 2011; Ioannidis et al., 2018). With the exception of excessive Internet use, these behaviours are not new to civilisation. Scientific recognition and scrutiny of the underlying processes in many cases, however, is new. DSM-5 lists kleptomania (compulsive stealing) in another category to addictions.

Murat Yücel

murat.yucel@monash.edu

1 Monash Institute of Cognitive and Clinical Neurosciences (MICCN), School of Psychological Sciences, and Monash Biomedical Imaging (MBI) Facility, Monash University, Melbourne, Australia

2 Anxiety and Obsessive-Compulsive Spectrum Disorders Research Program, Institute of Psychiatry, Federal University of Rio de Janeiro, Rio de Janeiro, Brazil

3 D'Or Institute for Research and Education (IDOR), Rio de Janeiro, Brazil

4 Department of Psychiatry, University of Cambridge, and Cambridge and Peterborough NHS Foundation Trust, Cambridge, UK
By contrast, compulsive sexual behaviour disorder (excessive sexual drive or excessive sex acts), compulsive buyingshopping disorder, and Internet gaming disorder, are not yet listed but appear to share remarkable parallels. Whether or not formally recognized, these disorders and syndromes are commonplace, cause considerable suffering, and thus merit scientific investigation and treatment. By necessity, most work in this special issue focuses on substance addictions and gambling because these disorders have been studied the most.

The reviews in this special issue of Neuropsychology Review highlight the opportunities and pitfalls in relation to how psychological, neuropsychological, pharmacological, technological and combination approaches can be used to target key cognitive-affective processes that cut across traditional diagnostic and clinical boundaries of substance and behavioural addictions. Following a recent consensus Delphi framework for addiction (Yücel et al., 2018), the issue has a particular focus on the domain of decision-making, which is likely to be important both in improving our understanding of aetiological processes mediating vulnerability and chronicity of different addictions, but also in terms of developing new intervention strategies and treatments.

Several papers in this issue focus on theoretical constructs and models for considering substance and behavioural addictions. While compulsivity is heavily implicated in addictions, this is a remarkably under-studied research area, particularly when contrasted to the well-studied topic of impulsivity. As Luigjes and colleagues note in their review of literature defining compulsive behaviour, compulsive problems are extremely common forms of psychopathology although no consensus yet exists regarding its definition, thereby hampering transdiagnostic research efforts. In a welcome advance to address these issues, Luigjes et al. describe findings from a review of literature, emphasising phenomenological, observational, and explanatory aspects. A working phenomenological definition is proposed for compulsive behaviour encapsulating: (1) feeling as if one needs to perform the behaviour, (2) the behaviour 
is repetitive, and (3) the individual is aware the behaviour is not consistent with their overall goal. Limitations in this approach are also discussed, including difficulty applying this definition across species. Other complementary approaches are considered, particularly an observational approach incorporating: (1) repetitive behaviour that is, (2) non-adaptive or inappropriate, leading to (3) functional impairment, and performed in a (4) habitual or stereotyped manner.

Of relevance to the latter observational definition of compulsivity, as well as to impulsivity, Lee et al. report findings from a comprehensive meta-review (i.e., meta-analysis of meta-analyses) of studies using relevant neurocognitive tasks in addictions. They report that alcohol dependence and gambling disorder were consistently associated with pervasive impulsivity and compulsivity deficits. Lee et al. find that other addictive disorders had been less comprehensively scrutinised to date, especially for compulsivity, and also that there appear to be some differences in neurocognitive profiles between classes of substance addiction. This meta-review supports the centrality of impulsivity and compulsivity in substance and behavioural addictions whilst also highlighting the need for more research into some disorders within this framework, disorders which are neglected. The authors also discuss how these neuropsychological measures of impulsivity and compulsivity can be used to inform clinical practice.

In the article by Rochat et al., the centrality of decisionmaking in understanding addictions is reviewed, including composite processes within a framework model. Starting with an historical angle, the paper describes the subsequent shift to a neurocognitively-grounded approach involving imbalance in a dual-process framework: an 'impulsive' system (implicating positive and negative reinforcement) and a 'reflective' system (prefrontal cortex and higher level executive functions). The authors then apply this model, and "open" it up for an extensive update to integrate the latest evidence, which includes interoreceptive processing and social processing, both of which are neglected in prior modelling frameworks of substance and behavioural addictions. Rochat et al. also provide a useful overview of measurement tools and paradigms that are available as probes of these decision-making cognitive systems.

The other focus of this special issue is treatments for the substance and behavioural addictions. Boffo et al. present findings from a novel individual patient data meta-analysis on the effects of Cognitive Bias Modification (CBM) for alcohol and smoking addictions. Using both a traditional frequentist and a Bayesian approach, they find that CBM had a small positive effect on cognitive bias and relapse rate, but not on reduction of substance use. Bayesian effects had very wide confidence intervals. The authors thus highlight the need for further high quality randomized controlled trials of standardised CBM protocols, before more substantive conclusions can be made as to whether or not CBM is as an effective behaviour change therapeutic intervention for addictions.
Several papers in this special issue consider candidate treatment options specifically to improve decision-making in addictions given its centrality to these disorders. Verdejo-Garcia et al. conducted a systematic review of neuropsychological interventions, finding evidence that Goal Management Training, Contingency Management plus Cognitive Behavioural Therapy, and Reality Therapy, had positive effects on aspects of decision-making in addiction. In particular, Goal Management Training (GMT) was found to have a moderate beneficial effect on cognitive control and emotional systems (the latter via mindfulness), while Contingency Management (CM) had similar beneficial effects on reward-related decision-making. Evidence for working memory training or standalone Cognitive Behavioural Therapy was mixed. Verdejo and colleagues indicate the need for rigorous trials to establish the efficacy of promising interventions and to examine whether decision-making effects generalize to global clinical outcomes. Chamberlain and Grant examine the potential use of medications to ameliorate decision-making deficits in addictions. Highlighting the value of decision-making deficits as candidate vulnerability markers for addiction, they first consider whether decision-making is objectively impaired in these disorders. They report that group case-control studies have identified decision-making deficits in substance use disorders, gambling disorder, kleptomania, and shop-lifting. These authors note the caveat that meta-analysis to confirm such deficits has so far only been reported for opioid use disorders. Pharmacological treatment evidence is surveyed for alcohol use disorder (as an archetypal substance addiction) and for behavioural addictions, with most studies focusing on global clinical outcomes rather than decision-making specifically. The authors consider the role of the dopamine system in decision-making deficits in addictions, both in the context of Parkinson's Disease (anti-dopaminergic medication as a cause of decision-making problems), and in terms of new therapeutic directions. The article links the decision-making deficits that have been found in addictions to related neural circuitry, implicating reductions in cortical thickness, and aberrant activation of fronto-striatal pathways. It is proposed that future clinical trials incorporate not only global symptom measures but also trans-diagnostic measures such as of decision-making.

Rolland et al. consider whether cognitive training programmes could be valuable as early intervention strategies for addictions. Focusing on data for substance use disorders, their meta-analysis revealed substance-specific associations between cognitive 'early impairments' (vulnerability markers) and early relapse or drop-out from treatment, indicating new and pragmatic treatment directions to avert such events. Lastly in terms of treatment related papers, Luigjes et al. comprehensively review the use of brain modulation in the treatment of addictions, including trans-cranial direct current stimulation, transcranial magnetic stimulation (TMS), functional imaging neurofeedback, and deep brain stimulation. They highlight 
promising data from initial controlled trials of transcranial magnetic stimulation and trans-cranial direct current stimulation studies as well as a lack of well-designed studies for EEGneurofeedback, the need for greater tailoring of neuromodulation to individual's neurocognitive profiles, and finally the need for investigation into deep brain stimulation and fMRI neurofeedback, these modalities being relatively new.

\section{References}

American Psychiatric Association. (2013). Diagnostic and statistical manual of mental disorders (5th ed.) (DSM-5). Arlington, VA: American Psychiatric Publishing.

Grant, J. E., Potenza, M. N., Weinstein, A., \& Gorelick, D. A. (2010). Introduction to behavioral addictions. The American Journal of Drug and Alcohol Abuse, 36(5), 233-241.

Ioannidis, K., Treder, M. S., Chamberlain, S. R., Kiraly, F., Redden, S. A., Stein, D. J., ... Grant, J. E. (2018). Problematic internet use as an age-related multifaceted problem: Evidence from a two-site survey. Addictive Behaviors, 81, 157-166.
Kalivas, P. W., \& Volkow, N. D. (2005). The neural basis of addiction: A pathology of motivation and choice. The American Journal of Psychiatry, 162(8), 1403-1413.

Mueller, A., Mitchell, J. E., Peterson, L. A., Faber, R. J., Steffen, K. J., Crosby, R. D., \& Claes, L. (2011). Depression, materialism, and excessive internet use in relation to compulsive buying. Comprehensive Psychiatry, 52(4), 420-424.

Yücel, M., Oldenhof, E., Ahmed, S., Belin, D., Billieux, J., BowdenJones, H., Carter, A., Chamberlain, S. R., Clark, L., Connor, J., Daglish, M., Dom, G., Dannon, P., Duka, T., Fernandez-Serrano, M. J., Field, M., Franken, I., Goldstein, R. Z., Gonzalez, R., Goudriaan, A., Grant, J. E., Gullo, M. J., Hester, R., Hodgins, D., Le Foll, B., Lee, R. S. C., Lingford-Hughes, A., Lorenzetti, V., Moeller, S. J., Munafo, M. R., Odlaug, B., Potenza, M. N., Segrave, R., Sjoerds, Z., Solowij, N., Van Den Brink, W., Van Holst, R. J., Voon, V., Wiers, R., Fontenelle, L. F. \& VerdejoGarcia, A. (2018). A transdiagnostic dimensional approach towards a neuropsychological assessment for addiction: An international Delphi consensus study. Addiction. https://doi.org/10.1111/add. 14424.

Publisher's Note Springer Nature remains neutral with regard to jurisdictional claims in published maps and institutional affiliations. 